Research Article

\title{
To Study efficacy and safety of citicoline in acute ischemic stroke
}

\author{
Nipunjot Grewal $^{\mathrm{a}, *}$, Geeta Sharma ${ }^{\mathrm{a}}$, Gurinder Mohan ${ }^{\mathrm{b}}$, Jaswinder Singh ${ }^{\mathrm{a}}$
}

${ }^{\mathrm{a} D e p a r t m e n t ~ o f ~ P h a r m a c o l o g y, ~}$ Sri Guru Ram Das Institute of Medical sciences and Research, Amritsar, India,

${ }^{\mathrm{b}}$ Department of Medicine, Sri Guru Ram Das Institute of

Medical sciences and Research, Amritsar, India

Received: 28 August 2012

Accepted: 14 September 2012

*Correspondence to:

Dr. Nipunjot Grewal,

E-mail:

dr_ngrewal@yahoo.co.in

\begin{abstract}
Background: Stroke is a medical emergency with mortality rate higher than most forms of cancer. Acute ischemic stroke is a complex entity with variable clinical manifestations depending on the site and extent of infarction. Besides standard treatment given to the patients, neuroprotection is being targeted to antagonize molecular events that lead to irreversible ischemic injury.

Methods: In this study, role of Citicoline in acute ischemic stroke was studied. It was open label study of 12 weeks duration undertaken in Medicine department (emergency unit) of Sri Guru Ram Das Institute of Medical Sciences and Research, Vallah, Amritsar. Total 40 patients were randomly divided into Group 1 and Group 2. Group 1 received standard treatment for acute ischemic stroke and Group 2 received citicoline in addition to standard treatment. Patients were assessed at admission and after every 24 hours till hospital discharge. Follow up of the patients was done at three weeks, six weeks and twelve weeks after discharge using National Institute of Health Stroke Scale (NIHSS), Modified Rankin Scale (MRS) and Modified Barthel Index (MBI). The data was statistically analysed using Mann Whitney test.

Results: No significant difference was found between two groups with respect to MRS and MBI score throughout the study period. Statistically significant improvement was seen in citicoline group on NIHSS score by $2^{\text {nd }}$ and $3^{\text {rd }}$ day of admission and then on $12^{\text {th }}$ week.

Conclusions: Citicoline was found to be safe but with no statistically significant difference in treatment outcome between two groups.
\end{abstract}

Keywords: CDP-choline, Ischemic penumbra, Neuroprotection

\section{INTRODUCTION}

Stroke, also sometimes called as 'brain attack' is defined as 'acute neurologic dysfunction of vascular origin with sudden (within seconds) or at least rapid (within hours) occurrence of symptoms and signs corresponding to involvement of focal areas in the brain'. ${ }^{1}$ Risk factors include advanced age, hypertension, previous stroke or transient ischemic attack, diabetes, hyperlipidemia, cigarette smoking, recreational drug abuse, heavy alcohol consumption and atrial fibrillation. Risk of stroke doubles for each decade after age of $55 .^{2}$ Stroke may be classified as ischemic or hemorrhagic with ischemic type contributing to maximum cases. Approximately $45 \%$ of ischemic strokes are caused by small or large artery thrombus, $20 \%$ are embolic in origin and rest have an unknown cause. Human brain comprises $2 \%$ of body weight, but requires $20 \%$ of total oxygen consumption. Five cardinal signs of stroke include weakness, speech impairement, vision impairement, headache and dizziness. ${ }^{3}$ The main goal in treating acute ischemic stroke is to preserve healthy brain tissue surrounding the blockage. This can be achieved by removing the blockage and restoring blood flow to the area, or by protecting the surrounding tissue. ${ }^{4}$ Aim of neuroprotection in acute ischemic stroke is to interrupt the propagation of these cascades in time to minimise permanent disability to the patient. Neuroprotection by citicoline has been described since 1978. Also called CDP choline, it reduces ischemic injury by stabilizing cell membrane and reducing free radical generation. Possible mechanisms include repair of neuronal membrane via increased synthesis of phosphatidyl choline, repair of damaged cholinergic neurons via potentiation of Acetyl choline production and reduction of free fatty acid build up at site of stroke induced neuronal damage. It is a water soluble compound with more than $90 \%$ bioavailability, rapidly absorbed and less than $1 \%$ excreted in faeces. Animal studies have shown that brain uptake of citicoline is only $0.5 \%$ of the oral dose, which can be increased to about $2 \%$ when this drug is given intravenously. ${ }^{5}$ Citicoline has been studied worldwide in both ischemic and hemorrhagic clinical 
stroke with excellent safety and possibly efficacy found in several trials. ${ }^{6}$ Besides its use in stroke patients, citicoline may be useful in conditions like head trauma of varying severity, cognitive disorders, Parkinson's disease, glaucoma and strabismus.

\section{METHODS}

This was a randomized, open label study of 12 weeks duration that was undertaken in Department of medicine (emergency unit) at Sri Guru Ram Das Charitable Hospital attached to Sri Guru Ram Das Institute of Medical Sciences and Research, Vallah, Amritsar. Total 40 patients of either sex fulfilling the inclusion and exclusion criteria and who reported to the emergency within 24 hours of onset of symptoms were included in the study. Patients were randomly divided into two groups of 20 patients each.

Group 1 received standard treatment of acute ischemic stroke i.e. Antiplatelets, ACE inhibitors, Statins, Decongestives and Anticoagulants, if necessary.

Group 2 were given citicoline in the dose of $1 \mathrm{~g}$ BD intravenous infusion dissolved in $100 \mathrm{ml}$ normal saline for 7 days in addition to standard therapy, followed by citicoline $500 \mathrm{mg}$ BD per oral for eight weeks. Patients were assessed clinically and through neurological examination were done at admission and regularly throughout their hospital stay. Follow up of the patients was done at three weeks, six weeks and twelve weeks after discharge using National Institute of Health Stroke Scale $(\mathrm{NIHSS})^{7}$, Modified Rankin Scale $(\mathrm{MRS})^{8}$ and Modified Barthel Index (MBI) ${ }^{9}$.

Patients of either sex more than 18 years of age with stroke onset of less than $24 \mathrm{hr}$ duration with a measurable focal deficit lasting for a minimum of $60 \mathrm{~min}$ were included in the study. All the patients were ambulatory and functionally independent before stroke and they had a CT/ MRI compatible with clinical diagnosis. All patients had Modified Rankin Scale $\leq 2$ before stroke and National Institute of Health stroke Scale $\geq 5$ at the time of presenting in the hospital. Written informed consent was taken from patients or their attendants before including them in the study.

Patients in coma or with severe coexisting terminal systemic illness or radiological evidence of brain tumor were excluded from the study. Also patients who required surgery within 24 hours or had drug addiction related disorders were not included.

The assessment was done using National Institute of Health Stroke Scale (NIHSS), Modified Rankin Scale (MRS) and Modified Barthel Index (MBI). Data was statistically analysed using Mann Whitney U test.

This study was conducted in accordance with the principles of good clinical practice and declaration of
Helsinki. The approval of institutional ethics committee was obtained.

\section{RESULTS}

The observations were recorded and tabulated as Mean \pm SD and analysed statistically. Table 1 gives baseline characteristics for both the groups. Twenty patients were enrolled in each of the patient groups. There was no significant difference noted in terms of patient age, sex, vascular territory involved and mean baseline NIHSS, MRS and MBI scores. Most of the patients had history of hypertension in the past i.e. $70 \%$ in group 1 and $90 \%$ in group 2, for which they were taking treatment irregularly. The incidence of stroke was more in patients who were on irregular treatment suggesting that patients who controlled the blood pressure adequately by taking treatment regularly have less chances of having stroke.

An improvement in chronological sequence has been observed in relation to their baseline values throughout the study in both the groups. On imaging, infarct was seen in MCA territory in most of the patients in both the groups. The changes in values of NIHSS, MRS and MBI are shown in Figure 1, 2 and 3 respectively. The decrease in NIHSS became significant by $2^{\text {nd }}$ and $3^{\text {rd }}$ day and again by $12^{\text {th }}$ week of treatment whereas decrease in MBI and MRS was statistically non-significant.

Throughout the study, no adverse event was observed which could be attributed to citicoline. Two patients out of twenty who took citicoline complained of disturbed sleep which might have been due to the disease as well.

Table 1: Baseline characteristics.

\begin{tabular}{|l|l|l|}
\hline & Group 1 & Group 2 \\
\hline Mean Age (years) & 60.65 & 61.7 \\
\hline Sex (Men) & $75 \%$ & $70 \%$ \\
$\begin{array}{l}\text { Hypertension } \\
\text { Diabetes Mellitus }\end{array}$ & $70 \%$ & $90 \%$ \\
$\begin{array}{l}\text { Vascular Territory } \\
\text { involved }\end{array}$ & & $30 \%$ \\
\hline ACA & $5 \%$ & $10 \%$ \\
\hline MCA & $80 \%$ & $60 \%$ \\
\hline PCA & $15 \%$ & $30 \%$ \\
\hline Baseline NIHSS & $15.15 \pm 4.64$ & $13.7 \pm 4.85$ \\
\hline $\begin{array}{l}\text { Baseline MRS } \\
\text { Baseline MBI }\end{array}$ & $3.9 \pm 0.55$ & $3.55 \pm 1.15$ \\
$\begin{array}{l}\text { Mean Serum } \\
\text { Cholesterol }\end{array}$ & $177 \pm 37.46$ & $178.35 \pm 33.90$ \\
$\begin{array}{l}\text { Mean Serum } \\
\text { Triglycerides }\end{array}$ & $155.55 \pm 42.86$ & $187.40 \pm 80.37$ \\
$\begin{array}{l}\text { Mean LDL } \\
\text { Mean VLDL }\end{array}$ & $104.05 \pm 32.28$ & $91.2 \pm 32.10$ \\
\hline
\end{tabular}




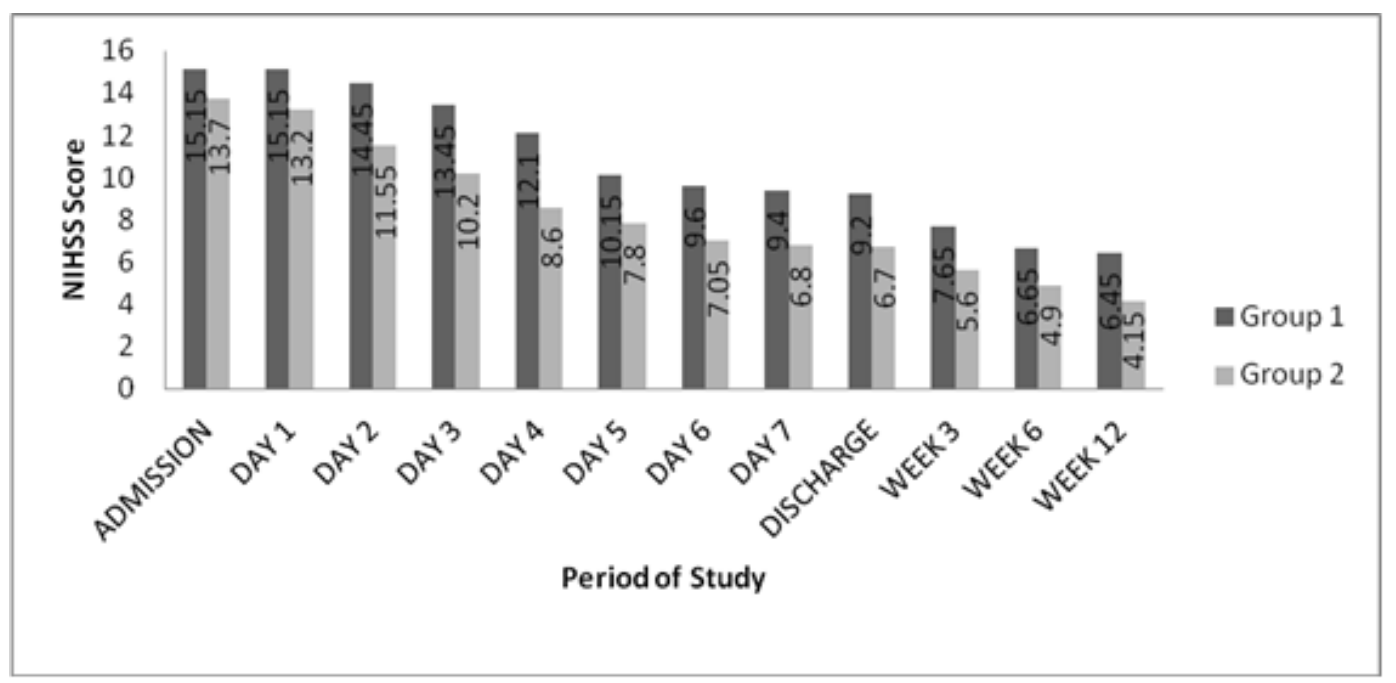

Figure 1: NIHSS score in Group 1 and Group 2.

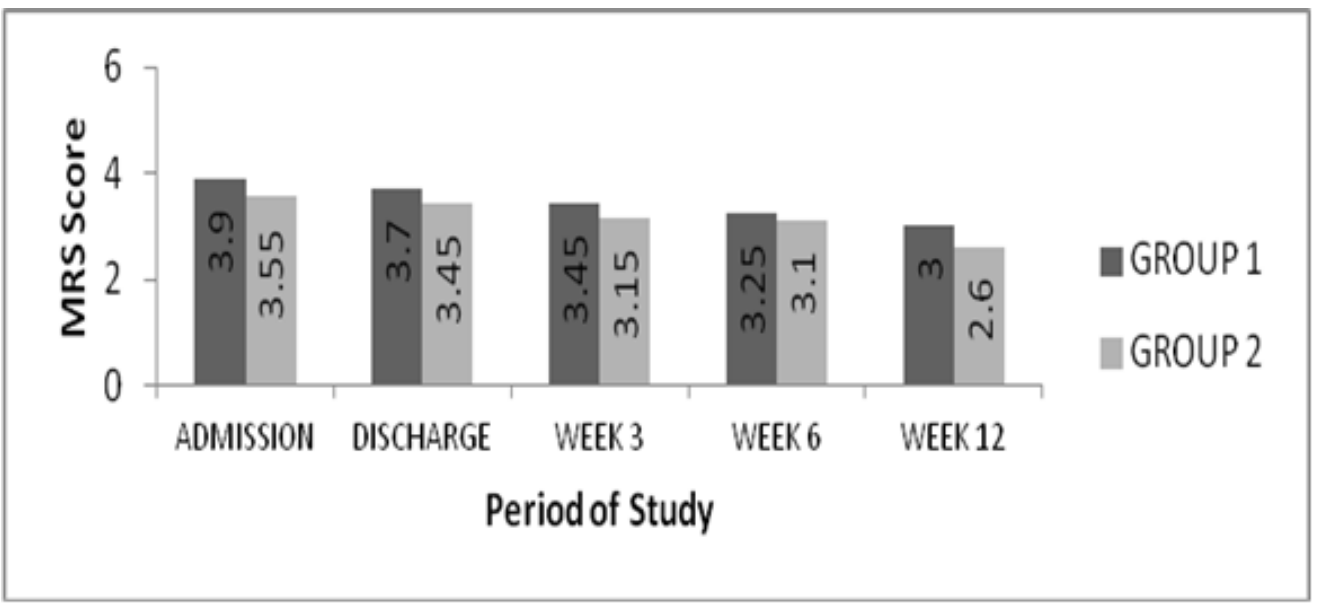

Figure 2: MRS score in Group 1 and Group 2.

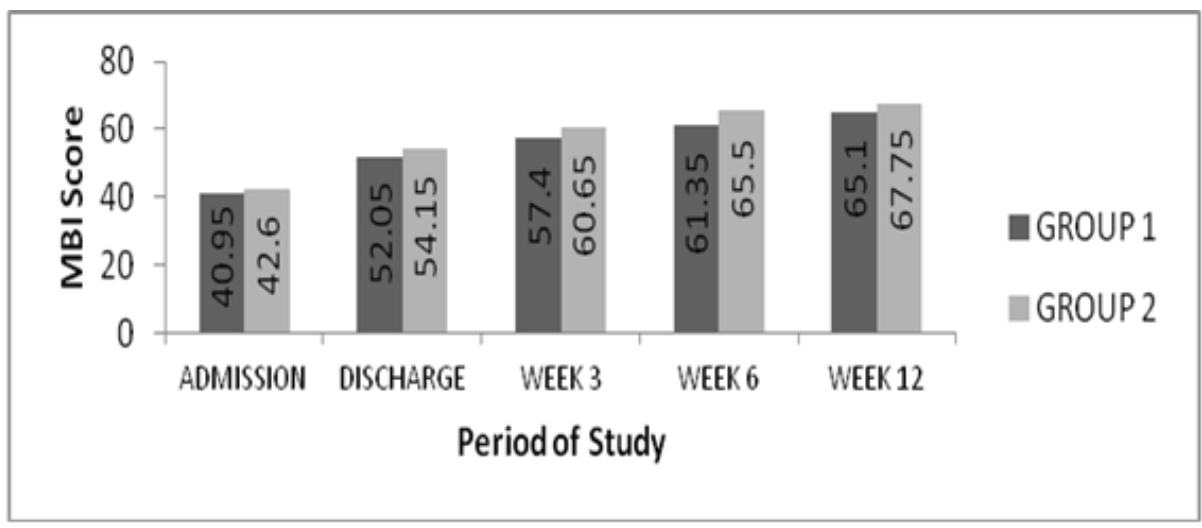

Figure 3: MBI score in Group 1 and Group 2. 


\section{DISCUSSION}

In Acute stroke which is one of the leading causes of mortality and morbidity worldwide may be attributed to various factors like changes in lifestyle, stress associated with biochemical alterations in the body. If the diagnosis of stroke is made in time and timely intervention done, it can save precious lives of many patients. Though it involves increased economic burden on the patient, yet treatment is far from satisfactory. Over the last few years, a huge number of compounds that interfere with biochemical mechanisms which mediate ischemic brain injury have been demonstrated to be neuroprotective in preclinical models of stroke. But drugs that survived safety trials and were studied in phase III clinical trials have so far failed to prove their efficacy. Citicoline or CDP-choline, a compound normally present in all the cells of the body, is both a neuroprotective when administered exogenously as well as an intermediate in membrane phosphatide biosynthesis.

This study found that citicoline can be administered safely to acute ischemic stroke patients with minimal side effects. In this study, $1000 \mathrm{mg}$ of citicoline given for 8 weeks significantly improved 12 week recovery as measured on acute assessment scale $(p<0.05)$ in comparison to patients who did not take citicoline. No significant improvement was seen on other measures which included Functional assessment (Modified Rankin Scale) and Outcome assessment scale (Modified Barthel Index). The baseline variables that were significantly different in both the groups were Serum cholesterol and Serum LDL values (higher in citicoline group). It is possible that some interaction between these parameters and recovery may have biased the data. Although baseline stroke severity did not vary between two groups, there was a trend towards higher values in citicoline group. Given the relatively small number of patients in both groups, this might have had an impact on overall efficacy assessment.

There was no statistical improvement in patients of both groups as assessed by MRS and MBI scales. This was in conformity with a study conducted by Clark et al who undertook a randomized efficacy trial of citicoline in 394 patients of acute ischemic stroke and compared a placebo with citicoline; $500 \mathrm{mg}$ orally for 6 weeks with 6 weeks post treatment follow up. There were no intergroup differences observed on analysis at 90 days including Barthel Index $\geq 95$ at 12 weeks. ${ }^{10}$

Similar results on NIHSS scale were observed by Clark et al also who compared the efficacy of citicoline with a placebo in their study. Dose of citicoline used was 1000 mg orally BD for 6 weeks with 6 weeks post treatment follow up period. Mean baseline NIHSS score were 14.5 for placebo and 13.9 for citicoline. The incidence and side effects were similar between two groups. ${ }^{11}$
Also in a drug surveillance study on 4191 patients of acute ischemic stroke conducted by Cho et al, oral citicoline (500 - $4000 \mathrm{mg} /$ day) was administered within less than 24 hours in 3736 patients (which formed early group) and later than 24 hours of stroke in 455 patients (in later group). Assessment was done using the similar scales as in the present study. The outcomes were improved after 6 weeks of therapy. Further improvement was observed in 125 patients who continued citicoline therapy for more than 12 weeks as compared to those who ended therapy at 6 weeks. Improvements were more significant in the higher dose group ( $\geq 2000 \mathrm{mg} /$ day). ${ }^{12}$

Goyas et al studied the effects of intravenous citicoline given in dose of $750 \mathrm{mg} /$ day for approximately 10 days within 48 hours of the stroke onset in a double blind, placebo controlled trial. Patients treated with citicoline were significantly more likely to be ambulatory at 90 days as compared to placebo treated patients as shown on quantified neurological assessment scale rating motor strength, muscular force, sensation, higher cortical function and ambulation. The drawback of this study was that it did not use currently accepted measures of neurological evaluation or functional outcome.

\section{CONCLUSIONS}

Efficacy of citicoline in treatment of acute ischemic stroke is limited as a neuroprotective agent, but it is very safe drug as per adverse effect profile.

\section{ACKNOWLEDGEMENT}

Department of Pharmacology and Medicine, Sri Guru Ram Das Institute of Medical Sciences and Research, Amritsar, Punjab, India.

\section{REFERENCES}

1. Goldstein M, Barnett HJM, Orgogozo JM, Sartorius N, Symon L, Vereshchagin NV. Recommendations on stroke prevention, diagnosis and therapy. Report of the WHO Task Force on Stroke and other Cerebrovascular Disorders. Stroke 1989;20(10): 1407-31.

2. Bamford J, Sandercock P, Dennis M, Burn J, Warlow C. A prospective study of acute cerebrovascular disease in the community: the Oxfordshire Community Stroke Project--1981-86. 2. Incidence, case fatality rates and overall outcome at one year of cerebral infarction, primary intracerebral and subarachnoid haemorrhage. J Neurol Neurosurg Psychiatry 1990 Jan;53(1):16-22.

3. Baldwin K, Orr S, Briand M, Piazza C, Veydt A, McCoy S. Acute Ischemic Stroke Update. Pharmacotherapy 2010 May;30(5):493-514.

4. Aminoff MJ. Nervous system disorders. In: McPhee SJ, Papadakis MA, Tierney LM. Current Medical Diagnosis and Treatment. 47ed. New York: McGraw Hill; 2008:837-96. 
5. Adibhatla RM, Hatcher JF. Citicoline mechanisms and clinical efficacy in cerebral ischemia. J Neurosci Res 2002 Oct 15;70(2):133-9.

6. WM Clark. Efficacy of citicoline as an acute stroke treatment. Expert Opin Pharmacother. 2009 Apr;10(5):839-46.

7. Brott T, Adams HP, Olinger CP, Marler JR, Barsan WG, Biller J et al. Measurements of acute cerebral infarction: a clinical examination scale. Stroke 1989;20:864-70.

8. Wilson JL, Hareendran A, Grant M. Improving the assessment of outcomes in stroke: use of a structured interview to assign grades on the modified Rankin Scale. Stroke 2002;33:2243-6.
9. Shah S, Vanclay F, Cooper B. Improving the sensitivity of the Barthel Index for stroke rehabilitation. J Clin Epidemiol 1989;42(8):703-9.

10. Clark WM, Williams BJ, Selzer KA, Zweifler RM, Sabounjian LA, Gammans RE. A randomized efficacy trial of citicoline in patients with acute ischemic stroke. Stroke 1999;30:2592-7.

11. Clark WM, Wechsler LR, Sabounjian LA, Schwiderski UE. A Phase III randomized efficacy trial of $2000 \mathrm{mg}$ citicoline in acute ischemic stroke patients. Neurology 2001;57(9):1595-602.

12. Cho HJ, Kim YJ. Efficacy and safety of oral citicoline in acute ischemic stroke: drug surveillance study in 4,191 cases. Methods Find Exp Clin Pharmacol 2009 Apr;31(3):171-6. 